\title{
REGIONALISMOS EN EL MARCO DE LAS RELACIONES INTERNACIONALES DEL SIGLO XXI
}

\author{
Pedro Manuel Rodríguez Suárez
}

Benemérita Universidad Autónoma de Puebla, México

http://dx.doi.org/10.5209/rev_NOMA.2012.v34.n2.40744

\begin{abstract}
Resumen.- Este artículo analiza el fenómeno del regionalismo y las transformaciones que ha tenido a través de la historia. Asimismo, indaga las variables que han jugado un papel fundamental en relación a la edificación de los regionalismos. Cabe resaltar que después de la Guerra Fría las agrupaciones regionales se han expandido por todo el mundo, en gran medida debido al fin de la confrontación ideológica, los procesos de democratización que tuvieron efecto en un gran número de países, los problemas transnacionales que afectan a las regiones, así como por los retos inherentes a la globalización. El objetivo cardinal de este artículo es el de evaluar las variables que propician la formación de los regionalismos, así como evaluar porqué algunas agrupaciones regionales son exitosas y otras fracasan. Para tal efecto, este artículo se fundamenta en la Teoría de Juegos y en la Teoría Neoinstitucional.
\end{abstract}

Palabras clave.- regionalismo, cooperación interregional, nuevo orden internacional, e integración regional.

\begin{abstract}
This article analyzes the phenomenon of regionalism and the transformations it has had throughout its history. It also investigates the variables that played a key role in building regionalism. It should be noted that after the Cold War regional groupings have expanded worldwide, largely due to the end of ideological confrontation, the processes of democratization that took effect in many countries of the world, transnational problems that affect regions, as well as the challenges inherent to globalization. The central objective of this paper is to evaluate the variables that promote the building of regional groupings as well as, assess why some regionalism are markedly successful and others fails. To this end, this article is based on game theory and neo-institutional theory
\end{abstract}

Keywords.- regionalism, interregional cooperation, new international order and regional integration.

\section{Introducción}

En la década de los ochenta y noventa convergieron una serie de factores que impactaron directamente en las agendas económicas, políticas y de seguridad de los Estados, entre los que resaltan: la tercera ola de transiciones hacia la democracia, el fin de la guerra fría, el acelerado proceso de globalización, el reconocimiento de los Estados en torno a las nuevas amenazas a su seguridad de carácter transnacional y multidimensional, así como el ascenso de los regionalismos.

El fin de la Guerra Fría transformó radicalmente las relaciones internacionales. En este orden de ideas, han surgido nuevas teorías que intentan comprender y definir el nuevo orden internacional de post Guerra Fría. Asimismo, ha sido necesario replantear el rol de la cooperación internacional e interregional, la importancia de los países emergentes, y la viabilidad de los organismos internacionales; tales como: la Organización de las Naciones Unidas (ONU), el Fondo Monetario Internacional (FMI), el Banco Mundial (BM), la 
Organización Mundial del Comercio (OMC), debido a que la gran mayoría de estos organismos ya no reflejan el actual status quo de las relaciones internacionales del siglo XXI. En adición a lo anterior, la prioridad actual de un número muy considerable de países es la de buscar nuevas formas de cooperación desde un punto de vista cuantitativo y cualitativo, y en particular desde un ámbito interregional.

Sin duda alguna, el regionalismo juega hoy en día un papel fundamental en las relaciones internacionales. Este fenómeno no es nada nuevo y tiene una fuerte tradición, cuyos orígenes provienen desde antes de la historia del capitalismo. Cabe resaltar que después de la Guerra Fría las integraciones regionales se han expandido por todo el mundo, en gran medida debido al fin de la confrontación ideológica, los procesos de democratización que tuvieron efecto en un gran número de países de África, América, Asia y Europa del Este, los nuevos dilemas de seguridad, la nueva interdependencia económica y política, así como por los retos inherentes a la globalización.

Por otro lado, la reciente propagación de los regionalismos demuestra la convicción empírica de los países en relación a que ya no tienen la capacidad individual para satisfacer sus necesidades, responder a los desafíos internacionales del siglo $\mathrm{XXI}$, proyectar sus intereses ante la comunidad internacional, solucionar problemas transnacionales, así como en incrementar su competitividad vis-á-vis otras regiones del mundo.

Es importante subrayar que el regionalismo, al menos desde su fundamento teórico, connota la antítesis de la autarquía y del unilateralismo, debido a que incorpora entre sus prioridades la promoción de la cooperación en detrimento de las acciones unilaterales.

1. Egresado de la licenciatura en Relaciones Internacionales por la Universidad Iberoamericana, Ciudad de México, diplomado en Estudios Europeos por el ITAM y doctor en Estudios de Integración Europea por la Universidad de Varsovia, Polonia. Actualmente es profesor-investigador de tiempo completo en la Benemérita Universidad Autónoma de Puebla (BUAP), México. Miembro del Sistema Nacional de Investigadores nivel 1-CONACYT. Sus líneas de investigación son: cooperación internacional, regionalismo y desarrollo regional. Coordinador y autor del libro: "América Latina: regionalización, cooperación internacional y problemas medio ambientales", publicado por la BUAP y la Universidad Autónoma de San Luis Potosí (2012); autor del libro: "Hacia una nueva Europa: la integración de los países de Europa Central y Oriental en la Unión Europea", editado por el FCE (2006). Ha efectuado estancias académicas y de investigación en Argentina, España, Polonia, Hungría y Uruguay. 
El regionalismo puede ser definido como una forma de cooperación internacional intermedia, que se establece por medio de la creación de alianzas y de la cooperación interregional entre países que por lo general comparten una misma región geográfica, aunque no siempre es así el caso. Asimismo, puede ser definido como una acción del Estado, e inclusive de la sociedad civil, así como de otros actores de las relaciones internacionales, cuya finalidad es la de promover la cooperación interregional. De igual manera, las integraciones regionales se fundamentan en valores, en un sentido de pertenencia e intereses compartidos, cuya finalidad es la de contrarrestar los múltiples retos que enfrentan los Estados desde una óptica regional. Los fundamentos para que se establezca el regionalismo, o como algunos autores lo Ilaman el "minilateralismo", radican en un interés común, similitud de sistemas económicos y políticos, complementariedad económica, afinidad de idiomas, cultura, tradición histórica y religión.

A pesar de lo que considera una gran parte de la opinión pública en relación a que no es posible establecer integraciones regionales cuando existen notorias asimetrías en términos de desarrollo económico e importantes diferencias culturales, la integración de Europa Occidental en el marco de la Unión Europea (UE) ha demostrado que sí es factible y que los resultados pueden ser altamente positivos, en particular vis-á-vis los países emergentes o menos desarrollados, los que a largo plazo resulta de gran beneficio para toda la región. Sin embargo, para maximizar los beneficios de la integración regional, es necesario que los países manifiesten una gran voluntad política en aras de profundizar su cooperación interregional, y establecer los mecanismos que institucionalicen dicha cooperación. Este fenómeno no ha sucedido en otras partes del mundo como en América del Norte con el Tratado de Libre Comercio de América del Norte (TLCAN), o bien, en Europa Oriental, con la Comunidad de Estados Independientes (CEI), (Clarkson, 2009 pp. 85-89).

Como marco teórico se utilizará la Teoría de Juegos, que presenta Karl Deuscht y la Teoría Neoinstitucional que sustenta Sven Steinmo. La primera plantea dos escenarios, el primero está relacionado al Juego de Suma Variable. En pocas palabras, los actores que deciden formar una integración regional establecen el contexto ad hoc para obtener beneficios equitativos y comunes. Duescht lo define como un juego de ganancias o beneficios mutuos. Por el contrario, en el Juego de Suma Zero, plantea que sólo uno o dos países obtienen los mayores beneficios en detrimento de los menos desarrollados. En este tipo de integraciones regionales el nivel de institucionalización y de cooperación interregional es muy limitado. La segunda teoría que utiliza este artículo es la Teoría Neoninstitucional. Desde la perspectiva de Steinmo, las instituciones son un grupo de reglas persistentes y conectadas que prescriben roles de comportamiento, constriñen la actividad y moldean las expectativas. Es decir, establecen las reglas del juego dentro de los regionalismos, así como su nivel de cooperación y muy probablemente definen las posibilidades de su éxito o fracaso, sin dejar de un lado, la voluntad política de los países para ampliar o limitar su cooperación.

Este artículo presenta tres objetivos fundamentales. Primero, analizar cómo fueron los regionalismos durante la Guerra Fría y cómo se han transformado 
después del conflicto ideológico Este-Oeste. Segundo, indagar las variables que han jugado un papel fundamental hacia la conformación de las agrupaciones regionales que se han establecido en las últimas décadas, así como analizar los diferentes niveles de integración que existen, y tercero, evaluar los factores que conllevan a que algunas integraciones regionales sean exitosas, mientras otras fracasan o permanecen en el olvido.

\section{MARCO TEÓRICO}

Este artículo se fundamenta en dos marcos teóricos que permiten analizar la esencia de los regionalismos del siglo XXI. Asimismo, ayudarán a comprender desde una perspectiva teórica los resultados que hasta el día de hoy han generado los regionalismos en diferentes partes del mundo. Es importante subrayar que ambos marcos teóricos sustentan la hipótesis que alude que a mayor grado de institucionalización y cooperación, los regionalismos tienen mejores posibilidades de éxito, mientras que los que presentan un bajo nivel de institucionalización y una cooperación relativamente limitada, obtienen pocos resultados y en muchas ocasiones tienden a desaparecer o permanecer en el olvido.

En este sentido, desde la perspectiva de la Teoría de Juegos que sustenta Karl Deuscth, en particular desde la óptica de la Teoría de Juegos de Suma Variable, todos los países que se integran en un regionalismo determinado obtienen grandes beneficios, en particular los países emergentes o menos desarrollados, quienes se favorecen del progreso de los países desarrollados. Sin embargo, esta teoría alude a que no siempre este es el caso y pueden presentarse escenarios totalmente inversos, como el Juego de Suma Zero. En este escenario, los países más desarrollados, obtienen los grandes beneficios en detrimento de los países emergentes. Este contexto frecuentemente se presenta en las Áreas de Libre Comercio (ALC) que poseen un mínimo nivel de integración y de cooperación interregional, así como de institucionalización. Asimismo, en las ALC no existen Fondos Estructurales y de Cohesión, por lo tanto, no hay mecanismos que auxilien a encontrar un equilibrio en términos de desarrollo económico y social.

Desde la óptica de la Teoría Neoinstitucional que sostiene Sven Steinmo, la cooperación interregional que está fuertemente institucionalizada connota mayores reglas persistentes y conectadas que prescriben roles de comportamiento, constriñen la actividad y moldean las expectativas. En pocas palabras, la institucionalización de la cooperación establece "las reglas del juego" que van a predominar en los regionalismos, así como el nivel de cooperación en términos cuantitativos y cualitativos. De igual manera, las instituciones, al ser parte del proceso dinámico de los regionalismos regulan el comportamiento de los países miembros. Asimismo, supervisan el escenario para que los países actúen de manera recíproca haciendo racionales sus interacciones. En este sentido, para la Teoría Neoinstitucional, el éxito de los regionalismos depende en gran medida de la edificación de las instituciones desde una perspectiva cualitativa y cuantitativa. 


\section{REGIONALISMOS DURANTE LA GUERRA FRÍA}

Un considerable número de especialistas de las relaciones internacionales coinciden en subrayar que los regionalismos se han expandido en el marco del nuevo orden (Stoessinger, 1994, p.32) en términos cualitativos y cuantitativos, y que actualmente las integraciones regionales son muy diferentes a las que existían en el statu quo ante, debido a un considerable número de factores.

Desde la perspectiva de Björn Hette, durante el conflicto ideológico que dividió al mundo por más de medio siglo, la gran mayoría de los regionalismos estaban subordinados a la lógica de la Guerra Fría, así como en la lucha por el poder mundial que mantenían las dos superpotencias que dominaban al mundo, Estados Unidos y la ex Unión de Repúblicas Socialistas Soviética (URSS). Ambas potencias mantenían un poder hegemónico en la mayoría de las agrupaciones regionales. En este sentido, la edificación de los regionalismos durante el conflicto Este-Oeste, estuvo fuertemente inspirada en la lucha ideológica, así como en las doctrinas de seguridad que imperaban en la política exterior de Moscú y de Washington, y en algunas partes del mundo como en América Latina, los regionalismos se establecían desde una perspectiva retórica y muchas veces hasta nacionalista, que trataban de contrarrestar la enorme influencia de Estados Unidos en el subcontinente latinoamericano.

En adición a lo anterior, los regionalismos de la era bipolar en la mayoría de las ocasiones no respondían a los intereses de los países que los conformaban, por el contrario predominaban los de las grandes potencias hegemónicas. Un ejemplo empírico sobre la esencia de estos esquemas de integración lo constituyeron el Pacto de Varsovia (PV), el Consejo de Ayuda Mutua Económica (CAME), la Organización de Estados Americanos (OEA), la Organización del Tratado del Sudeste Asiático (OTSA), la Organización del Tratado Central (OTC), el Tratado Interamericano de Asistencia Recíproca y la ANZUS (1), entre muchas otros (Bernatowicz, 1994, pp 27-32).

Es importante subrayar que el sistema de organizaciones regionales previsto por los arquitectos de la (ONU), alude en su Capítulo VIII, en su Carta Constitutiva que la finalidad de las agrupaciones regionales es la de propiciar la paz y la estabilidad por medio de la solución pacífica de las controversias regionales. A pesar de los anhelos de los arquitectos de la ONU, la gran mayoría de las agrupaciones regionales fueron utilizadas para maximizar los recursos de poder de las grandes potencias en el contexto de la lucha por el dominio mundial. Asimismo, los cinco miembros permanentes del Consejo de Seguridad, tales como, China, Estados Unidos, Francia, Rusia, y el Reino Unido, utilizaron al Consejo de Seguridad para profundizar sus rivalidades, lo que en muchas ocasiones paralizó su funcionamiento.

Aunque los regionalismos de la época de la Guerra Fría contaban con un amplio esquema de institucionalización, la "cooperación" estaba sujeta al Juego de Suma Zero, y los beneficios e intereses estaban supeditados a los de las dos superpotencias, en detrimento de los países periféricos que no tenían un campo de acción muy amplio para proyectar sus intereses (Rodríguez, 2006) . 


\section{REGIONALISMOS SIGLO XXI}

El fin de la Guerra Fría y el nuevo orden hexapolar han creado un escenario muy diferente al statu quo ante que prevaleció durante 1947 hasta 1991. Sin duda alguna, la conclusión del conflicto Este-Oeste dió origen al orden multipolar y, por consiguiente, al multilateralismo que ha permitido que los Estados posean una mayor libertad y campo de acción para proyectar su política exterior sin la interferencia de las superpotencias hegemónicas (Bernatowisz, et al, pp 59-62).

Asimismo, el fin de la hegemonía de los Estados Unidos en muchas esferas del poder mundial, tales como: la economía, la ciencia y la tecnología, así como en el comercio exterior, ha favorecido al resurgimiento de las potencias mundiales, continentales y regionales, que intentan incrementar su peso político y económico en sus respectivas zonas de influencia y en el concierto internacional, como bien lo ejemplifica el caso de Alemania y Francia en Europa Occidental, Rusia en Europa Oriental, Brasil en América del Sur, México en América Central y el Caribe, y China y Japón en Asia (Valencia, 2011, pp. 100-121)

En este sentido, Walt Roston se pregunta si la diversificación del poder mundial que caracteriza a las relaciones internacionales del siglo XXI, traerá consigo mayor estabilidad o inestabilidad hacia el escenario internacional. Para Roston, la multiplicidad del poder y la proliferación de los regionalismos promueven la estabilidad del orden internacional, debido a que fortalecen el multilateralismo en detrimento del unilateralismo, así como del poder hegemónico de una o varias superpotencias, tal y como sucedió durante la Guerra Fría. Otro factor que ilustra la naturaleza de los regionalismos del siglo XXI es que la geoeconomía posee un peso mayor que la geopolítica. En suma, la gran mayoría de los países otorga prioridad a las relaciones económicas internacionales, así como en aprovechar los beneficios que proporciona la cercanía geográfica y los mercados regionales.

Es importante resaltar que hoy en día los Estados que intentan conformar una agrupación regional, por lo general poseen los siguientes intereses:

- Incrementar su competitividad, desarrollo y su peso en el escenario internacional.

- Aprovechar al máximo sus ventajas competitivas en aras de atraer mayores y mejores inversiones. En este sentido, la cercanía geográfica, las similitudes culturales, y los gustos de los consumidores, juegan un papel sine qua non.

- Propiciar las condiciones económicas ad hoc que incrementen la calidad de vida de las personas, por medio de más y mejores empleos, y el libre acceso de bienes y servicios en los mercados regionales, así como por el incremento de la competencia en los mercados regionales. 
Cabe subrayar, que la tendencia actual hacia la globalización no representa un obstáculo hacia las integraciones regionales. En palabras de Marek Szczepánski (1998, p.45), "las integraciones regionales o el regionalismo es un fenómeno inherente de la globalización, cuya explicación puede ser asimilada desde una perspectiva sicológica, sociológica y hasta antropológica".

Por su parte, Ramón Tamames explica los factores que han sido determinantes hacia la conformación de los regionalismos en las ultimadas décadas:

1. La conformación de economías derivadas de la producción en gran escala.

2. La intensificación de la competencia dentro del nuevo mercado ampliado.

3. La posibilidad de abordar actividades que difícilmente podrían efectuar determinados países de manera individual o por separado, debido a la escasa dimensión de sus mercados nacionales, insuficiencia tecnológica, incapacidad financiera, etcétera.

4. El incremento del poder de negociación frente a terceros países o ciertas agrupaciones regionales.

5. La posibilidad de lograr, sobre la base de un rápido proceso de integración, la aceleración del desarrollo económico (no simplemente del crecimiento).

Por otro lado, un gran número de especialistas afirman que aunque no siempre es el caso, las integraciones regionales contribuyen a resolver los problemas transnacionales que enfrentan los Estados que comparten una misma región geográfica, tales como: degradación medioambiental, migración indocumentada, trata de personas, crimen organizado, y pérdida de competitividad vis-á-vis otros bloques regionales (Politi, 1997, pp, 2-10) En este mismo orden de ideas, los regionalismos al menos en América del Sur y en Europa Occidental han contribuido a fortalecer in situ la democracia, contrarrestar el unilateralismo de las grandes superpotencias y en mejorar sustancialmente las relaciones políticas entre los Estados, que anteriormente se percibían como rivales e inclusive como un peligro para su seguridad, tal y como lo evidencian las otrora relaciones entre Argentina y Chile, Argentina y Brasil, Alemania y Francia, Alemania y Polonia, India y Pakistán, lo que demuestra empíricamente algunos de los beneficios que pueden generar los regionalismos (Gálvez, 2011, pp 229-263)

Es importante señalar, que en el caso de Europa Occidental, la Unión Europea (UE) ha servido para inhabilitar cualquier posibilidad de conflicto bélico entre los países que conforman la UE por más de medio siglo. En adición, algunas de las economías de Europa Occidental anteriormente desbastadas por la II Guerra Mundial, se han transformado y colocado entre las más ricas, prósperas y competitivas del mundo, y el modelo de integración europeo ha contribuido para modernizar y desarrollar a países como España, Grecia, Irlanda, y Portugal, así como a los nuevos miembros de la UE, provenientes de Europa del Este, amén de los Fondos Estructurales y de Cohesión (Rodríguez et al., 2006, p.46). Asimismo, otro de los resultados de la integración de los países de Europa Occidental ha sido la abolición de las fronteras físicas entre los países, lo que ha contribuido a fortalecer las cuatro libertades de la UE: libre circulación de bienes, servicios, capitales y personas. 
Resulta necesario resaltar que no todos los regionalismos que se observan en el escenario internacional son iguales, ni tampoco han tenido los mismos resultados, y sería un grave error considerar que todos han sido exitosos. Por el contrario presentan enormes diferencias, desde la perspectiva de su nivel de integración, cooperación e institucionalización.

Al respecto, los regionalismos del siglo $X X I$ se pueden diferenciar desde las siguientes aristas:

a) Regionalismos cerrados y abiertos. Los regionalismos cerrados son los que comprenden entre sus miembros a países que se ubican en la misma región geográfica. Este tipo de regionalismos no están abiertos a países que se encuentran en otros continentes o regiones del mundo. En suma, su membresía está reservada al sentido de pertenencia geográfica, como es el caso de la UE o de la Unión Africana (UA). Por el contrario, los regionalismos abiertos son esquemas de integración cuya ubicación geográfica no es un elemento sine qua non para obtener su membresía, como lo ejemplifica la ANZUS.

b) Tratados preferenciales de comercio (TPC). Los TPC solo implican la disminución de aranceles entre los países miembros signatarios y un nivel de cooperación e institucionalización prácticamente inexistente.

c) Áreas de Libre Comercio (ALC). Las ALC connotan la eliminación de aranceles entre los países miembros. En este tipo de integración el nivel de cooperación interregional e institucionalización por lo general es muy bajo, tampoco existen instituciones supranacionales, ni mucho menos Fondos Estructurales y de Cohesión que promuevan el desarrollo equitativo entre los países miembros. Asimismo, en las ALC los países miembros no aplican un arancel externo común hacia terceros países. Tal es el caso del TLCAN, la Asociación Europea de Libre Comercio (AELC) y el G-2.

d) Uniones Aduaneras (UA). En las UA los estados miembros además de establecer un área de libre comercio y eliminar aranceles, aplican un arancel externo común hacia terceros países. La Unión Aduanera Centroamericana (UAC) y la Comunidad Andina (CAN), representa un claro ejemplo de este tipo de integración.

e) Mercados Comunes (MC). Los MC además de poseer las características de las ALC y las UA, establecen la libre circulación de factores productivos, especialmente mano de obra y capitales, tal es el caso del Mercado Común del Cono Sur (MERCOSUR). En este tipo de integraciones regionales, existe una cooperación e institucionalización mucho más amplia que en las ALC y las $U A$, y en algunos casos existen instituciones supranacionales y 
Fondos Estructurales y/o Regionales, cuyo objetivo es propiciar un equilibrio en términos de desarrollo entre los países miembros.

f) Uniones Económicas y Monetarias (UEM). Este tipo de integración comprende cuatro libertades: libre movimiento de personas, de bienes, capitales y servicios. Asimismo, existe una gran cooperación transfronteriza e instituciones supranacionales en donde los Estados ceden gran parte de su soberanía. Las UEM connotan la fase culminante de la integración incluyendo coordinación de políticas macroeconómicas y microeconómicas, y un sistema monetario común. Actualmente en algunos regionalismos como el de la UE, se estudia la posibilidad de ir más allá de la UEM, y convertir a Europa Occidental en una federación política, aunque esta idea no ha sido nada fácil de aceptar por un gran número de miembros, tales como: Dinamarca, Holanda, y el Reino Unido, así como por los nuevos miembros de la UE de Europa del Este.

\section{REGIONALISMOS ECONÓMICOS EN LA ERA DE LA GLOBALIZACIÓN}

Antes que iniciara la historia del sistema económico capitalista, algunos países ya habían establecido acuerdos en materia de integración económica. Sin embargo, como los Estados-nación constituían el núcleo básico del proceso de acumulación originaria del capital, los acuerdos de cooperación e integración económica regional eran sumamente escasos. Actualmente, esta situación se ha transformado radicalmente debido a que el Estado-nación representa un espacio económico muy reducido para los requerimientos del sistema capitalista, así como para las interacciones económicas del siglo XXI.

En este tenor, cada vez más se asume que el abaratamiento en los costos de producción, el incremento de la competitividad, y la generación de más y mejores empleos, se pueden alcanzar por medio de la cooperación internacional y, en particular de la cooperación interregional in situ que pueden establecer los países que comparten un mismo espacio geográfico. En este orden de ideas, la gran mayoría de los países del concierto internacional han incrementado sus esfuerzos en aras de ampliar su cooperación con los países vecinos con quienes comparten un determinado espacio geográfico. Desde la perspectiva económica, los objetivos son: la diversificación de los mercados en zonas geográficas claramente identificables, la erradicación de cualquier impedimento hacia el comercio interregional, así como de cualquier obstáculo que impida el libre flujo de bienes, servicios, capitales $y$, en algunos casos, hasta de personas.

En este contexto los regionalismos económicos de finales del siglo $X X$ e inicios del siglo XXI, nacen de la lógica de la distribución de la producción y de los recursos, de la revolución tecnológica y de los retos que enfrentan las regiones vis-á-vis el fenómeno de la globalización. Cabe subrayar que anteriormente los 
capitales internacionales tenían una participación internacional restringida. Con la apertura de los mercados internacionales, la revolución tecnológica, aunado a las transformaciones políticas y económicas que tuvieron efecto en África, Asia, América Latina y en Europa del Este a finales de la década de los ochenta y noventa, el incremento del comercio internacional ha tenido un impulso nunca antes visto en la historia de la humanidad. Este escenario ha sido decisivo hacia la regionalización de los mercados internacionales, que anteriormente se encontraban divididos por cuestiones políticas, económicas e ideológicas.

Aunque no hay una cifra exacta, se estima que entre un 50 y 60 por ciento del comercio mundial, circula entre los países que han establecido acuerdos de cooperación interregional. De acuerdo a la OMC, actualmente existen más de 100 acuerdos regionales alrededor del mundo. Cabe destacar que entre 1990 y 1994 se suscribieron el mayor número de acuerdos interregionales, lo que pone de manifiesto que las transformaciones mundiales que ocurrieron durante la década de los ochentas y noventas, tuvieron un efecto mayúsculo frente a la edificación de los regionalismos que se han establecido en los últimos años.

Como se mencionó con anterioridad, entre 1990 y 1994, se establecieron un gran número de acuerdos regionales, entre los que resaltan los siguientes: TLCAN (1994), MERCOSUR (1991), Consejo Económico Asia-Pacífico (APEC, 1989), Área Centro Europea de Libre Comercio (ACELC, 1992), Unión Monetaria y Económica de África Occidental (UMEAO, 1994), Unión Árabe del Magreb (UAM, 1989), Comunidad Económica Africana (CEA, 1991), Mercado Común para África del Sur y Oriental (COMESA, 1993), Sistema de Integración Centroamericano (SICA,1991), y la Asociación de Estados del Caribe (AEC, 1994).

Desde la perspectiva del GATT, los acuerdos regionales no son ilegales, ni tampoco constituyen un impedimento para el libre comercio. Al respecto, el artículo XXIV de esta organización, contempla la posibilidad de que dos o más naciones que se ubican en una misma región geográfica convengan establecer un tratamiento preferencial que no tendría que ser obligatoriamente extensivo hacia terceros países. Asimismo, estableció una serie de normas que debían ser acatadas por los países miembros, incluyendo la notificación que los países deberían hacer al GATT, en torno a la creación de los acuerdos económicos regionales.

Por otro lado, los acuerdos amparados en el artículo XXIV del GATT deberían cumplir con el requisito de cubrir todas las esferas comerciales y concretar una política comercial respecto a terceros países que no fuera más restrictiva que las políticas individuales previas a la suscripción de dichos acuerdos regionales. De igual manera el GATT estableció la siguiente normatividad:

- Los regionalismos económicos pueden instituirse siempre y cuando no discriminen a terceras partes.

- Los acuerdos regionales deben promover la liberalización comercial y no deben discriminar a terceros países. 
- Los regionalismos económicos no deben lesionar la liberalización de la economía mundial, por el contrario, deben promover la apertura de los mercados internacionales.

A pesar de las sugerencias emitidas por el GATT, en la praxis la gran mayoría de los regionalismos económicos son divisorios y pueden convertirse en un factor disfuncional y discriminatorio del comercio internacional. En este contexto, la mayoría de las agrupaciones económicas regionales que se han establecido son por esencia preferenciales, y en realidad segregan a terceros países frente al acceso de sus bienes y servicios a los mercados integrados debido a las "reglas de origen", que son utilizadas en la mayoría de las agrupaciones regionales o por estrategias como la Política Agrícola Común (PAC) de la UE.

En los primeros años de existencia del GATT, la observancia del artículo XXIV del GATT no presentaba grandes problemas, ya que de facto los miembros de este organismo acataban la idea en torno a la promoción del libre comercio. Asimismo, Estados Unidos poseía la hegemonía mundial de la economía capitalista, por lo que forzaba a las economías a abrir sus mercados al comercio internacional, por medio de instituciones como el FMI, el BM e inclusive el mismo GATT.

Con el surgimiento de las Comunidades Económicas Europeas (CEE) en la década de los 50, el artículo XXIV del GATT empezó a ser invocado cada vez más por los socios de las entonces CEE debido al enorme temor que expresaban en torno al ingreso de sus bienes y servicios en la economía comunitaria. Ante este contexto, otras regiones del mundo comenzarán a integrarse, entre otras cosas, debido a la sospecha que tenían sobre el nuevo proteccionismo europeo (Legler, 2010, pp 2-5)

Al respecto, en la década de los 60 surgieron instituciones como la Asociación Latinoamericana de Libre Comercio (ALALC, en 1960), el Pacto Andino (PA, en 1969), la Asociación de Libre Comercio del Caribe (CARIFTA, en 1967), la Asociación Europea de Libre Comercio (AELC, en 1960), la Asociación de Naciones del Sureste Asiático (ANSEA, en 1967), el Mercado Común Centroamericano (MCCA, en 1960), el Mercado Común Árabe (MCA, en 1964), y la Unión Económica y Aduanera de África Central (UEAAC, en 1964), entre otros.

Como se mencionó con anterioridad, la primera ola de regionalismos fue motivada, en gran medida, por la suscripción del Tratado de Roma por seis importantes economías europeas- Bélgica, Francia, Italia, Luxemburgo, los Países Bajos y la República Federal de Alemania-.

La segunda ola en torno a los procesos de regionalización, es decir, la que se produjo en la década de los 90 , ha constituido la más preocupante, debido a que las regiones del mundo se integraban como mercados ampliados e inclusive adquirían personalidad propia al punto de participar como bloques de países en las relaciones económicas internacionales, tal y como ha sido el caso de la UE en foros de suma importancia como el FMI, BM y GATT. 
Cabe resaltar, que hasta hoy en día, la UE es la única entidad regional que ha intentado incrementar su integración económica para convertirse tal vez en un futuro lejano en una entidad política y en algunos casos ha mantenido una postura común en materia de política exterior. Por otro lado, el MERCOSUR también ha propiciado que los países del Cono Sur de América hayan mantenido una posición conjunta en asuntos de carácter internacional, como por ejemplo en 1995, con el inicio de las negociaciones del Acuerdo Interregional de Cooperación UE-MERCOSUR, así como en la crisis política de Bolivia y Paraguay. Por otro lado, el TLCAN, la ASEAN, la ACELC, el Grupo de Visegrado, y el Acuerdo para el Establecimiento de Relaciones Económicas más Estrechas entre Australia y Nueva Zelanda, han contribuido a incrementar el diálogo político inter-bloques y la cooperación interregional, en un mundo en donde los regionalismos tendrán cada vez más relevancia y acciones concretas en la escena mundial.

\section{CONCLUSIONES}

Sin duda alguna los regionalismos del siglo $X X$ fueron radicalmente diferentes a los que existen actualmente. En los años de la Guerra Fría lo que imperaba era la lucha por el poder entre las dos potencias hegemónicas que se confrontaron ideológicamente por casi medio siglo. Desde esta perspectiva, la gran mayoría de las agrupaciones regionales, se edificaron desde la óptica del Juego de Suma Zero. Es decir, prevalecían los intereses y el poder hegemónico de los Estados Unidos y de la URSS, en detrimento de los países que pertenecían a su zona de influencia. Cabe destacar, que aunque existía un cierto grado de institucionalización y cooperación en la gran mayoría de dichas agrupaciones, sus efectos eran prácticamente nulos, debido a la enorme influencia que ejercían Washington y Moscú en los regionalismos del status quo ante. En adición, los pilares de los regionalismos del siglo $\mathrm{XX}$ fueron más geopolíticos que geoeconómicos.

Además, con el surgimiento de un nuevo orden internacional en1991, aparecen nuevas variables que transformarían al mundo radicalmente, tales como: la tercera ola de transiciones hacia la democracia, el fin de la Guerra Fría, el acelerado proceso de globalización, el reconocimiento de los Estados en torno a las nuevas amenazas sobre su seguridad de carácter transnacional y multidimensional, así como el ascenso de los regionalismos.

Desde el punto de vista funcional-estructural el regionalismo es una consecuencia, y al mismo tiempo, una manifestación del multilateralismo, así como del gran número de actores que intervienen en las interacciones internacionales del siglo XXI.

Como ha tratado de demostrar este artículo, los regionalismos juegan un papel fundamental en el siglo XXI, y pueden constituir una herramienta de desarrollo vis-á-vis los países emergentes o menos desarrollados, debido a que prevén la marginación de algunas naciones, fomentan el desarrollo, profundizan las relaciones de cooperación e incrementan el peso político de la región en el escenario internacional, así como su competitividad. 
Por otro lado, los regionalismos han contribuido a erradicar las rivalidades e incluso los antagonismos históricos que han prevalecido entre un gran número de países, tales como: Alemania y Francia; Alemania y Polonia; Reino Unido y Francia; Argentina y Brasil, y Argentina y Chile, así como entre la India y Pakistán. Sin embargo, para que este tipo de regionalismos tengan éxito deben ser edificados desde la óptica de la Teoría de Suma Variable y deben poseer un alto nivel de cooperación e institucionalización, así como una gran voluntad política con la finalidad de incrementar la cooperación interregional, tal y como lo postula la Teoría Neoinstitucional. Sin lugar a dudas, el regionalismo más exitoso que se percibe en la comunidad internacional es el de la UE, con su gran cooperación interregional, el alcance de su institucionalización, así como por los resultados que han tenido los Fondos Estructurales y de Cohesión en los países emergentes que se han integrado en la UE a través de la historia.

En los regionalismos en donde los resultados han sido menos exitosos, frecuentemente presentan un escenario radicalmente diferente. En suma, poco nivel de cooperación e institucionalización, así como una endeble voluntad política por parte de los países miembros para incrementar la cooperación y mucho menos la institucionalización. Desde el ámbito de la Teoría de Juegos de Suma Cero y de la Teoría Neoinstitucional, frecuentemente en este tipo de regionalismos existen uno o dos países que se favorecen más que los miembros menos desarrollados, y al no existir una cooperación relevante, ni tampoco instituciones que fomenten las reglas del juego, y por carecer de la voluntad política de sus miembros para ampliar la cooperación, por lo que por lo general los resultados que presentan son endebles. Este tipo de regionalismos puede ser ejemplificado con el caso del regionalismo en América del Norte con el TLCAN, en donde los resultados de la integración han sido sumamente exitosos en términos del gran incremento de las interacciones económicas, que según cifras oficiales se han triplicado. Sin embargo, sus contribuciones hacia el incremento de la competitividad de América del Norte, así como para el desarrollo de las regiones más pobres de América del Norte, han sido prácticamente inexistentes.

Asimismo, existen regionalismos que a pesar de que emergen con una gran voluntad política, por lo general presentan muy pocos resultados y se reducen a las buenas intenciones, así como a la retórica política. De acuerdo a la Teoría Neoinstitucional, su funcionalidad y operatividad son muy propensas al fracaso, al no existir instituciones que regulen sus respectivos modus operandi y cooperación. Este tipo de regionalismos se han proliferado en América Latina en las últimas décadas, como bien lo ejemplifica la Comunidad de Naciones de América Latina y el Caribe, y la Comunidad Andina de Naciones. Finalmente, existen otro tipo de regionalismos que aún operan como en la época de la Guerra Fría. Es decir, en donde existe el dominio de un sólo país vis-á-vis la gran mayoría, o bien, en donde los intereses nacionalistas o populistas prevalecen en detrimento de los intereses geoeconómicos, tal y como sucede con la Comunidad de Estados Independientes (CEI) y con la Alternativa Bolivariana para América Latina y el Caribe 
El panorama expuesto en este artículo nos obliga a reflexionar en torno a los regionalismos del siglo XXI y su papel en el actual orden internacional. Al respecto, aún no existen acuerdos entre los especialistas y se observan importantes diferencias en torno a sus posiciones sobre los efectos inherentes a las integraciones regionales. Para algunos, los regionalismos deben ser considerados como disfuncionales, debido a que sólo responden a los intereses de las empresas transnacionales, y son disfuncionales y discriminatorios hacia terceros países. Sin embargo, otra corriente de especialistas considera que las integraciones regionales constituyen el gran inicio de la integración mundial, en donde en un futuro todavía muy lejano no existirán fronteras para la libre circulación de bienes, servicios, capitales, y tal vez, de personas.

Sin duda alguna, la fortaleza y los buenos resultados de los regionalismos depende no sólo de la evolución de la economía mundial, de la globalización y las interacciones económicas entre sus miembros, sino también de la voluntad política de los Estados en aras de incrementar e institucionalizar dicha cooperación. Un claro ejemplo de esta afirmación lo constituye la UE y el TLCAN. La primera ha sido sumamente exitosa al haber logrado la reconstrucción de las economías europeas después de la II Guerra Mundial, la consolidación de la paz y de la democracia, así como por el establecimiento de una gran cooperación interregional por medio de instituciones supranacionales, así como por los Fondos Estructurales y de Cohesión. En relación a otros regionalismos como el TLCAN, ni Canadá, Estados Unidos y México, han conseguido ir más allá de un área de libre comercio. Al respecto, la Alianza para la Seguridad y la Prosperidad de América del Norte (ASPAN), formalmente instaurada en el 2005, tenía como objetivo fortalecer el regionalismo norteamericano y convertir a América del Norte en la región más competitiva del mundo, por medio del desarrollo de la infraestructura, y de una mayor institucionalización de la cooperación interregional. Sin embargo, a más de siete años de haberse establecido la ASPAN, esta alianza sigue siendo muy cuestionada y su funcionamiento ha sido poco relevante, debido a la falta de liderazgo de sus países miembros, el poco fomento que existe hacia la participación de la sociedad civil, así como por la endeble voluntad política que muestran los tres países con la finalidad de incrementar la cooperación e integración. Ante este escenario, los planteamientos de la Teoría de Juegos y la Teoría Neoinstitucional, dejan muy en claro que difícilmente los regionalismos pueden ser exitosos al prevalecer el Juego de Suma Zero y un bajo nivel de institucionalización, lo que explica el éxito, los resultados endebles, o en el peor de los casos, el fracaso, que se perciben en los regionalismos del siglo XXI. 


\section{BIBLIOGRAFÍA}

(1994) BERNATOWICZ, Grazyna, "Europe of Regions", The Polish Quarterly of International Affairs, ISSN 1230-4999, No.3, Varsovia, pp 27-32

(2010) BORBÓN, Josette, "La Alianza Bolivariana para los Pueblos de Nuestra América", Foregin Affairs Latinoamérica, ISSN 1665-1707, No.3, México, pp 3237

(2009) CLARKSON, Stephen, “¿Existe una América del Norte?", Revista Mexicana de Política Exterior,87, ISSN 0185-6022, México, pp 85-89

(2011) GALVÉZ, Yadira, "Una Comunidad de Seguridad y Defensa" en Alejandro Chanona (Cord.), Los Regionalismos frente a la Agenda de Seguridad Internacional, Miguel Ángel Porrúa, México, pp 229-263

(1996) HALIZAK, Edward,"Regionalism in International Relations", The Polish Quarterly of International Affairs, ISSN 1230-4999, 1996, Varsovia, p 234.

(2010) LEGLER, Toms, "El Perfil del Multilateralismo Latinoamericano", Foregin Affairs Latinoamérica, No.3, ISNN 1665 1770, México, pp 2-5

(1997) POLITI, Alessandro, "Nouveaux Risques Transnationaux et Securite Europeenne", Cahiers de Chaillot, No. 29, ISSN 1017-7574, Paris, pp 2-10

(2006) RODRIGUEZ, Pedro, Hacia una Nueva Europa: la integración de los países de Europa Central: La integración de los países de Europa Central y Oriental en la Unión Europea, Fondo de Cultura Económica, México.

(2011) RODRIGUEZ-SUAREZ, Pedro, "Brasil en el marco de la integración latinoamericana" en Carlos Figueroa (Cord.), ¿Posneoliberalismo en América Latina: los limites de la hegemonía neoliberal en la región?, Juan Pablos, México.

(1994) STOESSINGER, John, El Poderío de las Naciones: Política Mundial de Nuestro Tiempo, Ed. Gernika, México

(2011) VALENCIA, Alberto, Potencias Medias, Potencias Regionales en el Sistema Político Internacional de la Guerra Fría y Posguerra Fría. Propuesta de dos modelos teóricos, Ed.Universidad de Guadalajara, Guadalajara, pp 100121 